\title{
Cobertura vacinal da Pentavalente e da Estratégia de Saúde da Família*
}

\author{
Pentavalent Vaccine and Family Health Strategy Coverage
}

Cobertura de vacunación de la Pentavalente y del Programa Estrategia de Salud de la Familia

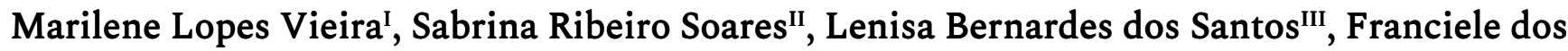 Santos Moreira ${ }^{\mathrm{IV}}$, Graciele Fernanda da Costa Linchv , Adriana Aparecida Paz ${ }^{\mathrm{VI}}$}

\begin{abstract}
Resumo: Objetivo: avaliar a cobertura vacinal da Pentavalente em menores de um ano nas regiões e capitais brasileiras e a cobertura da Estratégia de Saúde da Família (ESF). Método: estudo de série histórica de dados secundários provenientes de sistemas de gestão nacional de vacinas e da ESF, no período de 2014 a 2018, analisados por estatística descritiva. Resultados: as regiões brasileiras mantêm a cobertura vacinal da Pentavalente abaixo de 95\%, desde 2017. A abrangência da ESF não impactou significativamente no alcance da meta vacinal preconizada. Conclusão: as baixas coberturas vacinais aumentam o risco de reintrodução de doenças controladas. O acesso aos serviços de saúde precisa ser facilitado e a vacinação priorizada no planejamento das ações da equipe da ESF. A enfermagem tem condições de criar ações locais para reverter este cenário, com o apoio dos gestores para intensificar campanhas e combater as causas que têm prejudicado o alcance das metas.
\end{abstract}

Descritores: Cobertura vacinal; Vacinas; Atenção Primária à Saúde; Saúde da Família; Enfermagem

Abstract: Objective: to evaluate Pentavalent vaccination coverage in children under one year old in the Brazilian regions and capitals and the coverage of the Family Health Strategy (FHS). Method: a study of a historical series

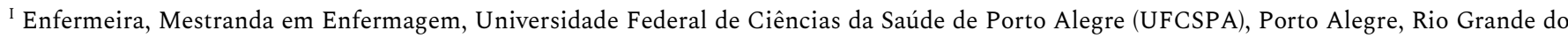
Sul, Brasil. E-mail: marilenelv991747701@gmail.com ORCID: https://orcid.org/0000-0002-2353-0974

II Enfermeira, Mestre em Enfermagem, Instituto de Cardiologia do Rio Grande do Sul, Porto Alegre, Rio Grande de Sul, Brasil. E-mail: sabrinarsoares@gmail.com ORCID: https://orcid.org/0000-0001-9521-827X

III Enfermeira, Mestre em Enfermagem, Instituto de Cardiologia do Rio Grande do Sul, Porto Alegre, Rio Grande do Sul, Brasil. E-mail: lenisas@gmail.com ORCID: https://orcid.org/0000-0002-5098-5042

IV Graduanda de Enfermagem, Universidade Federal de Ciências da Saúde de Porto Alegre (UFCSPA), Porto Alegre, Rio Grande do Sul, Brasil. E-mail: franmoreira.3132@gmail.com ORCID: https://orcid.org/0000-0002-8163-9257

V Enfermeira, Doutora em Enfermagem, Professora do Departamento de Enfermagem e do Programa de Pós-Graduação em Enfermagem, Universidade Federal de Ciências da Saúde de Porto Alegre (UFCSPA), Porto Alegre, Rio Grande do Sul, Brasil. E-mail: gracielelinch@ufcspa.edu.br ORCID: https://orcid.org/0000-0002-8802-9574

VI Enfermeira, Doutora em Enfermagem, Professora do Departamento de Enfermagem e do Programa de Pós-Graduação em Enfermagem, Universidade Federal de Ciências da Saúde de Porto Alegre (UFCSPA), Porto Alegre, Rio Grande do Sul, Brasil. E-mail: adrianap@ufcspa.edu.br ORCID: https://orcid.org/0000-0002-1932-2144
}

* Extraído do trabalho da disciplina "Sistemas de Informação em Saúde", Programa de Pós-Graduação em Enfermagem, Universidade Federal de Ciências da Saúde de Porto Alegre, 2019. 
with secondary data from national vaccine management systems and the FHS, from 2014 to 2018, analyzed by descriptive statistics. Results: the Brazilian regions have maintained Pentavalent vaccination coverage below 95\% since 2017. The scope of the FHS did not significantly impact on the achievement of the recommended vaccination target. Conclusion: low vaccination coverage increases the risk for the reintroduction of controlled diseases. Access to the health services needs to be facilitated and vaccination prioritized when planning the actions of the FHS team. Nursing is able to create local actions to reverse this scenario, with the support of the managers to intensify campaigns and combat the causes that have hindered the achievement of the goals.

Descriptors: Vaccination Coverage; Vaccines; Primary Health Care; Family Health; Nursing

Resumen: Objetivo: evaluar la cobertura de vacunación de la Pentavalente en menores de un año en las regiones y capitales de Brasil y la cobertura de la Estrategia de Salud de la Familia (ESF). Método: estudio de serie histórica de datos secundarios provenientes de sistemas de administración nacional de vacunas y de la ESF, en el período de 2014 a 2018, analizados por medio de estadística descriptiva. Resultados: las regiones de Brasil mantienen un nivel de cobertura de vacunación de la Pentavalente por debajo del 95\% desde el año 2017. La cobertura de la ESF no tuvo un efecto significativo para alcanzar la meta de vacunación recomendada. Conclusión: los bajos índices de cobertura de vacunación aumentan el riesgo de reintroducción de enfermedades controladas. Debe facilitarse el acceso a los servicios de salud y se debe priorizar la vacunación en la planificación de las acciones del equipo del programa ESF. El área de Enfermería tiene las condiciones necesarias para diseñar acciones locales a fin de revertir esta situación, con el apoyo de los administradores para intensificar campañas y combatir las causas que han perjudicado el alcance de las metas.

Descriptores: Cobertura de Vacunación; Vacunas; Atención Primaria de Salud; Salud de la Familia; Enfermería

\section{Introdução}

A vacinação é uma tecnologia imunobiológica disponível no sistema de saúde brasileiro, ofertada na rede de Atenção Primária à Saúde (APS). O uso desta tecnologia, conforme a Sociedade Brasileira de Imunizações (SBIm) e o Ministério da Saúde (MS), traz diversos benefícios à população pela capacidade de evitar mortes prematuras, adoecimento e internações por doenças imunopreveníveis, além de permitir a erradicação de doenças de alcance mundial como a varíola e a poliomielite. ${ }^{1-2}$

O Programa Nacional de Imunizações (PNI) do Brasil foi criado em 1973, ofertando gratuitamente à população brasileira as vacinas recomendadas pela Organização Mundial de Saúde (OMS). No Brasil, o PNI consegue oferecer os imunobiológicos nos mais distintos locais, como as comunidades ribeirinhas da floresta amazônica. ${ }^{1}$ No contexto brasileiro, a agência reguladora responsável por aprovar as vacinas desenvolvidas no decorrer dos longos processos 
de pesquisa é a ANVISA (Agência Nacional de Vigilância Sanitária), cujo papel fundamental na rigorosa avaliação e inspeção dos imunobiológicos garante a segurança da população. ${ }^{2}$

De acordo com o MS, o indicador de cobertura vacinal é um importante instrumento de gestão para a tomada de decisão em todas as esferas do governo, já que sinaliza o crescimento ou redução de doenças imunopreveníveis e reemergentes. ${ }^{3}$ A redução nas taxas de cobertura vacinal vem preocupando a comunidade científica e o governo, visto que, para a imunização ser considerada eficaz no bloqueio de doenças, deve-se completar o esquema vacinal com o número de doses recomendadas para cada tipo de imunobiológico assim como garantir coberturas homogêneas. Algumas causas para a diminuição da cobertura podem ser a insuficiência programada de alguns imunobiológicos na rede da APS, a divulgação de notícias falsas, o horário de atendimento das unidades de saúde e a hesitação vacinal. ${ }^{4}$

A hesitação vacinal ocorre quando os indivíduos atrasam a vacinação ou se recusam receber as doses de alguns tipos de imunobiológicos. Trata-se de um conjunto de comportamentos que podem estar influenciados por movimentos antivacinas, fazendo com que algumas pessoas posterguem a vacinação baseadas em convicções que justificam tal tomada de decisão..$^{5-6}$ Já a recusa vacinal ocorre quando o indivíduo nega todo e qualquer tipo de imunobiológico pelos mais variados motivos: princípios filosóficos ou religiosos, aspectos socioculturais, baixa percepção do risco de doenças, questionamentos sobre a eficácia das vacinas e orientação médica. ${ }^{5}$

Atualmente, o movimento antivacina ganha notoriedade devido à mobilização da sociedade contra a vacinação compulsória. Este movimento acredita que: o sistema imunológico da criança é incapaz de processar todo o número de antígeno aplicado; a vacinação causa doenças autoimunes; e a imunização desenvolvida ao se adquirir a doença é mais eficaz e segura do que a vacina. ${ }^{5} \mathrm{~A}$ propagação de informações falsas em mídias sociais reforça tais ideias, o que aumenta a insuficiência do bloqueio de doenças imunopreveníveis. 
As vacinas são formuladas a partir de agentes infecciosos ou de engenharia genética que, ao serem introduzidos no organismo, simulam a invasão de um agente infeccioso e estimulam o organismo a produzir anticorpos, criando a memória imunológica capaz de responder positivamente quando se entra em contato com o verdadeiro agressor. ${ }^{7}$ Uma importante vacina conjugada em um único imunobiológico e presente no calendário vacinal da criança é a Pentavalente, que protege contra a difteria, o tétano, a coqueluche, a hepatite B e o Haemophilus influenzae $b$. O esquema básico de aplicação é realizado com três doses: aos dois, aos quatro e aos seis meses de idade, sendo que a recomendação do MS delimita que as coberturas sejam superiores a 95\%. ${ }^{3}$

A nível mundial, existe um plano global de ação para vacinação (2011-2020) que prevê que todos os países alcancem cobertura vacinal maior ou igual a $90 \%$ em todas vacinas. A OMS e o Fundo das Nações Unidas para a Infância (UNICEF) estão envolvidos neste monitoramento e preconizam as ações para melhoria das coberturas vacinais de forma a se atingir a meta. Mundialmente, a vacina DTP (Difteria, Toxóide Tetânico e Coqueluche) apresentou cobertura de 84\% em 2010 e alcançou $86 \%$ em 2018, o que representa um discreto crescimento de $2,38 \%$. A cobertura da terceira dose da DTP é o principal indicador de eficácia de um programa de imunizações. ${ }^{8}$

A equipe de enfermagem é a responsável pela sala de vacinas e por todo o processo de armazenamento, preparo e aplicação dos imunobiológicos na APS, além do controle da cobertura vacinal, busca de faltosos e os registros eletrônicos e manuais adequados, medidas que garantem assim a qualidade dos imunobiológicos até o usuário final. ${ }^{9}$ Cabe aos auxiliares e técnicos em enfermagem a execução das atividades de rotina, colocando em prática todas as normativas técnicas e orientações do PNI, enquanto aos enfermeiros é atribuído o papel de responsabilidade técnica pela sala de vacinas, supervisão, educação continuada e implementação de ações para o alcance da cobertura vacinal. ${ }^{10-11}$

A Estratégia de Saúde da Família (ESF) é considerada a principal ferramenta para a expansão da cobertura da APS no território brasileiro. O cálculo de cobertura da ESF (cESF) é 
realizado por meio da multiplicação do número de equipes por 3.450 usuários, dividindo-se o resultado pela população estimada. Mundialmente, os países utilizam o número de usuários cadastrados e atribuídos a uma equipe ou a um médico. ${ }^{12}$

Partindo-se do cálculo de abrangência da ESF associado à cobertura vacinal em um mesmo território, torna-se possível observar: a implementação de novas equipes de ESF pelo gestor municipal; as ações para reduzir a ocorrência de doenças imunopreveníveis; e a qualidade dos serviços ofertados. A meta de vacinação é um importante indicador a se comparar com a ocorrência de doenças imunopreveníveis na população, bem como a qualificação do processo de trabalho da equipe de saúde. ${ }^{4}$ Nesse sentido, a equipe da ESF que atende os usuários em seu território conhece o perfil populacional e estende o cuidado da unidade de saúde ao domicílio. Desta maneira, possui condições para estabelecer ações que acolham e valorizem a manutenção do esquema vacinal completo. ${ }^{9}$ Depreende-se que quanto maior o indicador da cESF no município, mais qualificado é o processo de trabalho da equipe de saúde, na medida em que assim é possível que se desenvolvam ações pertinentes que se configuram em estratégias para a redução de doenças, destacando-se entre elas as imunopreveníveis.

Face ao exposto sobre a importância da imunização, o crescimento de doenças imunopreveníveis e reemergentes no território nacional e a adoção da ESF como modelo prioritário para ofertar saúde à população brasileira - vinculando o usuário a uma equipe de saúde - instigou-se a questão de pesquisa: "Como são as coberturas vacinais do imunobiológico Pentavalente em menores de um ano nas regiões e capitais brasileiras, e como se apresenta a cESF nesses locais?” O objetivo do presente estudo é avaliar a cobertura vacinal da Pentavalente (cvP) em menores de um ano nas regiões e capitais brasileiras e a cESF. 


\section{Método}

Trata-se de um estudo epidemiológico, de série histórica, realizado por meio de dados secundários gerados no registro das salas de vacinas da rede de APS e lançados no Sistema de Informação do Programa Nacional de Imunizações (SI-PNI), que é gerenciado pelo PNI e MS. O acesso aos dados foi disponibilizado pelo Departamento de Informática do Sistema Único de Saúde (DATASUS). Já os dados de cESF são oriundos do Sistema de Cadastro Nacional de Estabelecimento de Saúde (SCNES) e do Instituto Brasileiro de Geografia e Estatística (IBGE), que foram extraídos do Sistema de Informação e Gestão da Atenção Básica (e-Gestor AB). ${ }^{13-14}$

Em relação à cobertura vacinal, os critérios de seleção no DATASUS foram: cobertura vacinal, área geográfica (Brasil, regiões e capitais), imunobiológico Pentavalente no período de 2014 a 2018. A coleta de dados ocorreu entre outubro e dezembro de 2019, de acordo com as variáveis definidas pela disponibilidade no DATASUS. Em relação à ESF, os critérios selecionados no e-Gestor $A B$ foram: capitais brasileiras e percentual de cESF no mesmo período. $O$ caminho percorrido no e-Gestor $A B$ para a seleção das variáveis foi: relatórios públicos, histórico de cobertura de atenção básica e seleção das opções de consulta. Determinou-se tal intervalo de tempo devido ao fato de este apresentar os dados atualizados no momento da coleta dos mesmos nos dois sistemas.

Os dados foram salvos em planilhas de Microsoft Excel 2016 e analisados no mesmo programa pela estatística descritiva, utilizando-se a frequência absoluta e relativa. Os resultados são apresentados por meio de tabela para ilustrar a cobertura vacinal do imunobiológico e as coberturas de saúde da família nas capitais brasileiras. Por tratar-se de dois bancos de dados de domínio público, não foi necessário submeter esta pesquisa ao Comitê de Ética em Pesquisa. De qualquer forma, esta investigação respeita os preceitos éticos com base na Resolução nº 466/2012, do Conselho Nacional de Saúde. ${ }^{15}$ 
7 | Vieira ML, Soares SR, Santos LB, Moreira FS, Linch GFC, Paz AA

\section{Resultados}

A Tabela 1 apresenta a cobertura vacinal do imunobiológico Pentavalente administrado em crianças menores de um ano de idade, na Região Norte e suas capitais e a cESF, entre de 2014 a 2018.

Tabela 1 - Cobertura vacinal da Pentavalente (cvP) em crianças menores de um ano e cobertura da Saúde da Família (cESF), na Região Norte e capitais, no período de 2014 a 2018.

\begin{tabular}{|c|c|c|c|c|c|c|}
\hline Região/Capitais & & 2014 & 2015 & 2016 & 2017 & 2018 \\
\hline Norte & $\mathrm{cvP}^{*}$ & 86,50 & 85,08 & 78,24 & 72,63 & 71,14 \\
\hline \multirow[b]{2}{*}{ Belém } & $\mathrm{cvP}$ & 84,07 & 52,31 & 71,46 & 62,08 & 58,95 \\
\hline & $\mathrm{cESF}^{* *}$ & 22,38 & 22,73 & 24,62 & 23,80 & 23,61 \\
\hline \multirow[b]{2}{*}{ Boa Vista } & $\mathrm{cvP}$ & 79,60 & 98,71 & 92,29 & 97,54 & 76,17 \\
\hline & cESF & 58,43 & 55,88 & 58,67 & 57,07 & 56,37 \\
\hline \multirow[b]{2}{*}{ Macapá } & $\mathrm{cvP}$ & 72,54 & 87,69 & 101,11 & 57,77 & 57,67 \\
\hline & cESF & 58,97 & 63,96 & 61,63 & 38,91 & 52,38 \\
\hline \multirow[b]{2}{*}{ Manaus } & $\mathrm{cvP}$ & 86,74 & 98,61 & 96,23 & 76,66 & 74,09 \\
\hline & cESF & 31,90 & 33,40 & 30,19 & 26,51 & 29,77 \\
\hline \multirow{3}{*}{ Palmas } & $\operatorname{cvP}$ & 93,46 & 104,23 & 84,16 & 77,02 & 82,96 \\
\hline & & & & & & \\
\hline & cESF & 82,71 & 83,73 & 90,16 & 99,88 & 99,87 \\
\hline \multirow{3}{*}{ Porto Velho } & $\operatorname{cvP}$ & 97,28 & 96,85 & 97,17 & 105,79 & 107,86 \\
\hline & & & & & & \\
\hline & cESF & 54,00 & 53,77 & 55,98 & 51,62 & 51,58 \\
\hline \multirow[b]{2}{*}{ Rio Branco } & $\mathrm{cvP}$ & 66,02 & 87,59 & 78,84 & 78,42 & 67,46 \\
\hline & cESF & 50,14 & 56,01 & 58,12 & 58,79 & 56,53 \\
\hline
\end{tabular}

Fonte: SI-PNI (2019) e MS/SAPS/Departamento de Atenção Básica - DAB (2019)

Notas: ${ }^{*} \operatorname{cvP}$ - Cobertura vacinal da Pentavalente; ${ }^{* *}$ CESF - Cobertura da Saúde da Família.

Das cinco regiões brasileiras, a Região Norte (Tabela 1) foi a que apresentou a menor cvP (71,14\%) de forma global; porém, este mesmo indicador foi maior nas capitais Boa Vista, Manaus, Palmas e Porto Velho. Destaca-se a capital Porto Velho, onde a cvP aumentou em 
10,58\% ao se compararem os dados de 2014 e 2018, e a cESF se reduziu em 2,42\%. Em contraposição, as capitais dessa Região que apresentaram declínio na cobertura vacinal da Pentavalente e aumento da cESF foram, respectivamente, Palmas $(10,50 \%$ e 17,16\%) e Belém (25,12\% e 1,23\%). As demais capitais da Região Norte apresentaram o declínio na cvP e da cESF, respectivamente: Boa Vista (3,43\% e 2,06\%), Manaus (12,65\% e 2,13\%) e Macapá (14,87\% e 6,59\%).

A capital Porto Velho obteve as melhores coberturas vacinais da Região Norte por todo o período, permanecendo acima da meta preconizada em todos os anos estudados. Cabe registrar que, nesta capital, ocorreu a redução de cESF em 2,42\%, o que demonstra que o alcance da meta vacinal não está diretamente relacionado a este modelo de atenção à saúde. A outra capital, Rio Branco, embora não tenha os melhores indicadores das coberturas, apresentou um crescimento de $1,44 \%$ na cvP e $6,39 \%$ na cESF.

Nas capitais Macapá e Belém, observou-se um declínio da cvP que, em 2018, permaneceu em torno de 58\%. A cESF nestas capitais se contrapõe, sendo que em Belém houve um modesto crescimento, ao passo que em Macapá houve uma redução neste indicador nos cinco anos avaliados. Portanto, é possível inferir que a baixa cobertura vacinal pode ter relação com a cESF nestas unidades federativas. A Tabela 2 apresenta a cobertura vacinal do imunobiológico Pentavalente administrado em crianças menores de um ano de idade, na Região Nordeste e suas capitais e a cESF, no período de 2014 a 2018.

Tabela 2 - Cobertura vacinal da Pentavalente (cvP) em crianças menores de um ano e cobertura da Saúde da Família (cESF), na Região Nordeste e capitais, no período de 2014 a 2018.

\begin{tabular}{ccccccc}
\hline Região/Capitais & & $\begin{array}{c}2014 \\
\mathbf{\%}\end{array}$ & $\begin{array}{c}\mathbf{2 0 1 5} \\
\mathbf{\%}\end{array}$ & $\begin{array}{c}\mathbf{2 0 1 6} \\
\mathbf{\%}\end{array}$ & $\begin{array}{c}\mathbf{2 0 1 7} \\
\mathbf{\%}\end{array}$ & $\begin{array}{c}\mathbf{2 0 1 8} \\
\mathbf{\%}\end{array}$ \\
\hline Nordeste & $\mathrm{cvP}^{*}$ & 93,06 & 95,93 & 86,21 & 81,02 & 89,47 \\
Aracaju & $\mathrm{cvP}$ & 87,21 & 93,81 & 64,34 & 66,75 & 76,97 \\
& $\mathrm{cESF}$ & 76,81 & 74,11 & 70,79 & 64,40 & 65,32 \\
Fortaleza & $\mathrm{cvP}$ & 86,99 & 104,99 & 133,41 & 83,69 & 124,90
\end{tabular}


9 | Vieira ML, Soares SR, Santos LB, Moreira FS, Linch GFC, Paz AA

\begin{tabular}{|c|c|c|c|c|c|c|}
\hline & cESF & 42,61 & 46,83 & 48,39 & 48,12 & 46,32 \\
\hline \multirow{2}{*}{ João Pessoa } & $\mathrm{cvP}$ & 92,23 & 83,56 & 78,18 & 70,30 & 85,84 \\
\hline & cESF & 79,45 & 78,65 & 81,55 & 83,26 & 82,14 \\
\hline \multirow{2}{*}{ Maceió } & $\mathrm{cvP}$ & 77,18 & 78,87 & 76,14 & 66,79 & 79,31 \\
\hline & cESF & 29,47 & 29,51 & 29,24 & 28,78 & 28,03 \\
\hline \multirow{2}{*}{ Natal } & $\mathrm{cvP}$ & 74,79 & 80,66 & 77,19 & 44,29 & 100,28 \\
\hline & cESF & 47,53 & 46,08 & 41,54 & 33,19 & 38,64 \\
\hline \multirow{2}{*}{ Recife } & $\mathrm{cvP}$ & 90,30 & 96,29 & 93,80 & 84,87 & 90,02 \\
\hline & cESF & 53,25 & 51,94 & 54,01 & 56,24 & 55,68 \\
\hline \multirow{3}{*}{ Salvador } & $\mathrm{cvP}$ & 90,42 & 95,13 & 87,31 & 83,85 & 78,09 \\
\hline & & & & & & \\
\hline & cESF & 22,61 & 25,97 & 27,78 & 27,70 & 27,19 \\
\hline \multirow{2}{*}{ São Luís } & $\mathrm{cvP}$ & 77,95 & 87,20 & 85,13 & 76,47 & 62,74 \\
\hline & cESF & 33,86 & 35,51 & 32,93 & 34,99 & 34,73 \\
\hline \multirow{2}{*}{ Teresina } & $\mathrm{cvP}$ & 84,78 & 76,35 & 76,69 & 63,69 & 75,37 \\
\hline & cESF & 98,44 & 99,67 & 99,36 & 99,57 & 100,00 \\
\hline
\end{tabular}

Fonte: SI-PNI (2019) e MS/SAPS/Departamento de Atenção Básica - DAB (2019)

Notas: ${ }^{*}$ cvP - Cobertura vacinal da Pentavalente; ${ }^{* *}$ CESF - Cobertura da Saúde da Família.

$\mathrm{Na}$ Tabela 2, as capitais Fortaleza, Natal e Recife apresentam o indicador de cvP superior ao indicador da Região Nordeste $(89,47 \%)$ em 2018. A capital cearense - Fortaleza - apresentou crescimento da cvP $(37,91 \%)$ e da ESF (3,71\%), enquanto na capital Aracaju, observou-se o declínio de ambos indicadores (10,24\% e 11,49\%) ao se compararem os anos de 2014 e 2018 . As capitais Natal e Maceió apresentaram aumento da cvP, sendo respectivamente 25,79\% e 2,13\%, mas uma redução da cESF (8,89\% em Natal, e 1,44\% em Maceió). As demais capitais nordestinas apresentaram declínio da cvP e ampliaram a cESF na mesma comparação e na mesma ordem dos indicadores: Recife (0,28\% e 2,43\%), João Pessoa (6,39\% e 2,69\%), Teresina (9,41\% e 1,56\%), Salvador (12,33\% e 4,58\%) e São Luís (15,21\% e 0,87\%). 
A capital Fortaleza demonstrou os melhores resultados de cvP, alcançando índices acima de 100\% em 2015, 2016 e 2018, enquanto a capital potiguar - Natal - apresentou dados bem divergentes em relação à cvP, tendo no ano de 2017 o pior resultado da Região Nordeste com $44,29 \%$, e, em $2018,100,28 \%$, dado que aponta que houve incentivos e trabalho na capital para melhoria da cobertura vacinal. No entanto, a cESF em 2017 na capital era de 33,19\%, o que, com incentivos, elevou-se para 38,64\%; porém, em comparação a 2014 e 2018, constatou-se o declínio desse indicador. Apesar do discreto crescimento da cESF, o mesmo pode ter contribuído positivamente para a melhora da meta vacinal, somando-se com as demais ações em saúde realizadas.

As capitais Aracaju, João Pessoa, Maceió, São Luís e Teresina mantiveram a cvP ao longo do período avaliado sempre abaixo da meta de 95\%, destacando-se Maceió com o indicador em uma constante variando de $66,79 \%$ a $79,31 \%$. A capital piauiense - Teresina - apresentou a redução na cvP na comparação dos anos, e, por outro lado, observou-se que a cESF em 2018 atingiu a totalidade de $100 \%$ de seu território. A Tabela 3 apresenta a cobertura vacinal do imunobiológico Pentavalente administrado em crianças menores de um ano de idade, na Região Centro-Oeste e suas capitais e a cESF, no período de 2014 a 2018.

Tabela 3 - Cobertura vacinal da Pentavalente (cvP) em crianças menores de um ano e cobertura da Saúde da Família (cESF), na Região Centro-Oeste e capitais, no período de 2014 a 2018.

\begin{tabular}{|c|c|c|c|c|c|c|}
\hline Região/Capitais & & 2014 & 2015 & 2016 & 2017 & 2018 \\
\hline \multirow[t]{2}{*}{ Centro-Oeste } & $\mathrm{cvP}^{*}$ & 101,62 & 95,17 & 99,95 & 83,56 & 87,48 \\
\hline & $\mathrm{cvP}$ & 92,60 & 66,43 & 140,25 & 84,66 & 86,26 \\
\hline \multicolumn{7}{|l|}{ Brasília } \\
\hline & $\mathrm{cESF}^{* *}$ & 28,25 & 29,01 & 29,03 & 30,77 & 43,29 \\
\hline & $\mathrm{cvP}$ & 91,84 & 98,74 & 99,96 & 93,14 & 93,16 \\
\hline \multicolumn{7}{|l|}{ Campo Grande } \\
\hline & cESF & 36,54 & 36,28 & 36,25 & 35,99 & 47,65 \\
\hline & cvP & 86,73 & 91,39 & 86,64 & 81,16 & 73,60 \\
\hline \multicolumn{7}{|l|}{ Cuiabá } \\
\hline
\end{tabular}


11 | Vieira ML, Soares SR, Santos LB, Moreira FS, Linch GFC, Paz AA

$\begin{array}{llllll}\operatorname{cvP} & 87,76 & 83,46 & 77,53 & 72,77 & 81,08\end{array}$

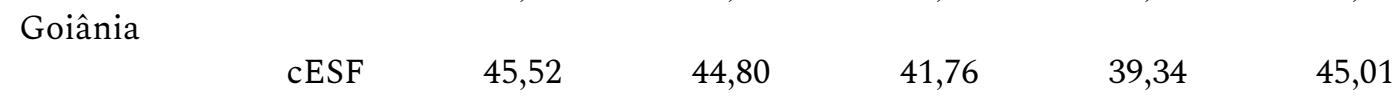

Fonte: SI-PNI (2019) e MS/SAPS/Departamento de Atenção Básica - DAB (2019).

Notas: ${ }^{*}$ cvP - Cobertura vacinal da Pentavalente; ${ }^{* *}$ CESF - Cobertura da Saúde da Família.

A capital Campo Grande destacou-se com uma cvP mais homogênea, tendo resultados acima de $90 \%$ em todos os anos analisados, sendo a única da Região Centro-Oeste (Tabela 3), em 2018 , cujo indicador $(93,16 \%)$ é superior ao regional $(87,48 \%)$. Ainda nesta capital, tal indicador mostrou um discreto crescimento de 1,32\% na comparação de 2014 e 2018. Os incentivos para ampliação da cESF fizeram com que o indicador atingisse 47,65\%.

As demais capitais, Brasília e Cuiabá, apresentaram declínio na cvP ao se avaliarem os anos de 2014 e 2018, sendo 6,34\% e 13,13\% respectivamente. Em relação à cESF, observou-se que em Brasília houve um incremento de $15,04 \%$ no indicador, e em Cuiabá, o aumento foi de 4,81\%. Entretanto, na capital Goiânia, este indicador se manteve constante ao longo do período estudado (45\%). A Tabela 4 apresenta a cobertura vacinal do imunobiológico Pentavalente administrado em crianças menores de um ano de idade, na Região Sudeste e suas capitais e a cESF, no período de 2014 a 2018.

Tabela 4 - Cobertura vacinal da Pentavalente (cvP) em crianças menores de um ano e cobertura da Saúde da Família (cESF), na Região Sudeste e capitais, no período de 2014 a 2018.

\begin{tabular}{|c|c|c|c|c|c|c|}
\hline Região/Capitais & & $\begin{array}{c}2014 \\
\%\end{array}$ & $\begin{array}{c}2015 \\
\%\end{array}$ & $\begin{array}{c}2016 \\
\%\end{array}$ & $\begin{array}{c}2017 \\
\%\end{array}$ & $\begin{array}{c}2018 \\
\%\end{array}$ \\
\hline Sudeste & $\mathrm{cvP}^{*}$ & 96,19 & 99,13 & 91,36 & 87,74 & 92,25 \\
\hline \multirow{3}{*}{ Belo Horizonte } & cvP & 57,24 & 85,23 & 94,86 & 84,10 & 90,06 \\
\hline & & & & & & \\
\hline & $\mathrm{cESF}^{\star *}$ & 76,11 & 78,87 & 78,76 & 77,60 & 77,15 \\
\hline \multirow{3}{*}{ Rio de Janeiro } & cvP & 96,92 & 99,48 & 104,50 & 108,50 & 97,64 \\
\hline & & & & & & \\
\hline & cESF & 42,11 & 43,04 & 51,56 & 62,63 & 60,99 \\
\hline \multirow[b]{2}{*}{ São Paulo } & cvP & 91,45 & 94,77 & 76,25 & 77,03 & 92,18 \\
\hline & CESF & 3308 & 3167 & 3391 & 3454 & 3622 \\
\hline Vitória & cvP & 109,08 & 93,11 & 105,58 & 94.98 & 95,42 \\
\hline
\end{tabular}


Cobertura vacinal da Pentavalente e da Estratégia de Saúde da Família | 12

$$
\begin{array}{llllll}
\text { CESF } & 73,22 & 74,06 & 72,00 & 72,33
\end{array}
$$

Fonte: SI-PNI (2019) e MS/SAPS/Departamento de Atenção Básica - DAB (2019).

Notas: ${ }^{*} \mathrm{cvP}$ - Cobertura vacinal da Pentavalente; ${ }^{* *} \mathrm{cESF}$ - Cobertura da Saúde da Família.

A Região Sudeste (Tabela 4) manteve a cobertura vacinal acima de 90\%, exceto no ano de 2017 (87,74\%). Rio de Janeiro e Vitória apresentaram os melhores indicadores de cvP nos anos avaliados, chegando a 108,50\% no Rio de Janeiro (2017) e 109,8\% em Vitória (2014). Em relação à cESF, as cidades do Rio de Janeiro e São Paulo apresentaram um crescimento de 18,88\% e 3,14\% respectivamente.

A capital paulista apresentou uma queda na cvP em 2016 e 2017, seguida de uma melhora na cobertura em 2018 alcançando um indicador superior a 92\%. Em relação à cESF, esta capital tem o pior indicador da Região Sudeste. Entretanto, observou-se que ocorreu uma discreta ampliação da cESF (3,14\%) entre 2014 e 2018. A Tabela 5 apresenta a cobertura vacinal do imunobiológico Pentavalente administrado em crianças menores de um ano de idade, na Região Sul e suas capitais e a cESF, no período de 2014 a 2018.

Tabela 5 - Cobertura vacinal da Pentavalente (cvP) em crianças menores de um ano e cobertura da Saúde da Família (cESF), na Região Sul e capitais, no período de 2014 a 2018.

\begin{tabular}{|c|c|c|c|c|c|c|}
\hline Região/Capitais & & $\begin{array}{c}2014 \\
\%\end{array}$ & $\begin{array}{c}2015 \\
\%\end{array}$ & $\begin{array}{c}2016 \\
\%\end{array}$ & $\begin{array}{c}2017 \\
\%\end{array}$ & $\begin{array}{c}2018 \\
\%\end{array}$ \\
\hline Sul & $\mathrm{cvP}^{*}$ & 97,36 & 98,40 & 91,80 & 87,14 & 89,52 \\
\hline \multirow{2}{*}{ Curitiba } & cvP & 90,71 & 95,93 & 96,01 & 88,12 & 93,56 \\
\hline & $\mathrm{cESF}^{* *}$ & 43,96 & 42,94 & 41,32 & 39,27 & 34,86 \\
\hline \multirow{2}{*}{ Florianópolis } & cvP & 86,37 & 83,71 & 90,54 & 10,58 & 77,62 \\
\hline & cESF & 90,57 & 95,37 & 98,79 & 96,00 & 76,28 \\
\hline \multirow{2}{*}{ Porto Alegre } & cvP & 82,46 & 78,86 & 71,59 & 75,90 & 74,39 \\
\hline & cESF & 38,35 & 41,63 & 45,00 & 46,33 & 53,87 \\
\hline
\end{tabular}

Fonte: SI-PNI (2019) e MS/SAPS/Departamento de Atenção Básica - DAB (2019).

Notas: *cvP - Cobertura vacinal da Pentavalente; ${ }^{* *}$ cESF - Cobertura da Saúde da Família. 
A Tabela 5 da Região Sul revela que as capitais Florianópolis $(8,75 \%)$ e Porto Alegre (8,07\%) apresentaram declínio na cobertura vacinal em comparação aos indicadores atingidos em 2014 e 2018. A capital Curitiba, em 2018, obteve o indicador de cvP 93,56\%, superando o indicador da Região Sul (89,52\%). Embora Curitiba apresente a melhor cvP em todos os anos avaliados, observa-se a redução da cESF de 9,10\%. No ano de 2017, Florianópolis apresentou uma das piores coberturas vacinais do Brasil (10,58\%). Este indicador tão baixo decorreu da falha no envio dos dados durante a implantação do Sistema de Informações do Programa Nacional de Imunizações (SIPNI) no período de 2016 a 2017.

A Região Centro-Oeste apresentou a melhor cvP em 2014 e 2016, a Região Nordeste atingiu a meta apenas em 2015, e as Regiões Sul e Sudeste o fizeram nos anos de 2014 e 2015. Os dados indicam que a cvP foi inferior a 95\% em todas as regiões brasileiras, desde 2017, variando de $71,14 \%$ a $92,25 \%$. A Região Norte manteve a meta sempre inferior ao preconizado e com a menor cobertura em 2018. No Brasil, reduziu-se em 6,41\% a cvP em 2018 na comparação com o ano de 2014. Este declínio foi diferente em todas as regiões brasileiras: Norte 14,36\%, CentroOeste $14,14 \%$, Sul 7,84\%; Sudeste 3,94\%, e Nordeste 3,59\%.

\section{Discussão}

No Brasil, a hesitação vacinal vem prejudicando a vacinação, visto que, na década de 90, as coberturas vacinais brasileiras passavam de 95\%, fato que possibilitou o controle e erradicação de algumas doenças infectocontagiosas, comprovando o sucesso do PNI. ${ }^{16}$ Com o passar do tempo e o avanço das redes de comunicação e mídias sociais, propagaram-se diversas notícias falsas sobre a vacinação, o que pode ter contribuído para a queda nas coberturas. ${ }^{7}$

No ano de 2016, a OPAS forneceu ao Brasil o certificado de eliminação da circulação do vírus do sarampo. Nos anos seguintes, em decorrência da baixa cobertura vacinal da Tríplice Viral (que protege contra Sarampo, Caxumba e Rubéola) e dos movimentos migratórios de 
países vizinhos, houve a reintrodução do sarampo e registro de surto em 2018, com mais de mil casos confirmados, o que levou à perda da certificação. Tal fato confirma que a baixa cobertura vacinal é um risco para a saúde da população. ${ }^{17}$

Assim, o controle das doenças imunopreveníveis pode ser alcançado, desde que os gestores e profissionais de saúde assumam o compromisso por meio de uma rede de serviços de saúde organizada e eficiente associada ao sistema de vigilância competente que seja capaz de desencadear rápidas ações em saúde. ${ }^{16}$ Entretanto, acredita-se que diversos fatores possam contribuir para as reais coberturas vacinais, tais como: o acesso e o horário de atendimento dos serviços de saúde; a hesitação vacinal por medo da dor ou desconforto na aplicação da vacina; a quantidade de imunobiológicos e doses a serem aplicados; a repreensão dos pais/responsáveis diante do atraso vacinal; e outros. ${ }^{18}$

Entre os fatores anteriormente mencionados, verifica-se que a repreensão que pais ou responsáveis temem receber da equipe de saúde por conta de eventuais atrasos na vacinação das crianças certamente pode contribuir para a hesitação vacinal e/ou reforçar esta conduta. Portanto, é importante entender este sentimento causado pela estigmatização e trabalhar para a desconstrução deste comportamento, ressaltando-se a importância da vacinação para proteção das crianças. ${ }^{17}$ Como os pais ou responsáveis cada vez mais têm buscado informações sobre os mais diversos assuntos, entre eles a saúde, nas redes sociais como o Facebook, este meio de comunicação é muito útil para divulgar informações sobre saúde e estimular a vacinação; entretanto, essa facilidade de acesso também pode provocar um efeito diferente do desejado e assim contribuir para o aumento da hesitação vacinal, pois, infelizmente, o movimento antivacina veicula diversas informações falsas sobre vacinas que são repassadas sem uma certificação do conteúdo. ${ }^{17,19-20}$

Logo, as autoridades governamentais precisam antever, vigiar e organizar ações para combater a divulgação de notícias falsas, denominadas de Fake News, devendo sempre 
responder às eventuais dúvidas a serem esclarecidas, para garantir assim a promoção e a proteção da população. Diante da velocidade de propagação das informações falsas, o governo brasileiro enfrenta o combate das Fake News disponibilizando o número +55 61-99289-4640 para atender a população via aplicativo de WhatsApp. Este recurso digital está disponível para smartphone, sendo possível enviar e receber mensagens de áudio, vídeos, fotos, entre outras possibilidades. O objetivo do MS é que a população verifique a veracidade da informação antes de compartilhá-la, e faz o alerta quanto ao risco à saúde de se divulgar e acreditar em informações incorretas. ${ }^{21}$

Por meio das pesquisas, obtém-se um melhor entendimento do que se passa na identificação e solução de eventuais problemas. Por exemplo, ressalta-se que as vacinas combinadas são um importante recurso para minimizar o número de aplicações, já que uma única vacina, como a Pentavalente, pode proteger contra várias doenças, além de reduzir os custos e melhorar a adesão dos pais e responsáveis. ${ }^{22}$

Outro estudo realizado em município do Noroeste do Estado do Rio Grande do Sul, em 2017, verificou o declínio nos indicadores da cobertura vacinal com a plena cobertura de equipes de ESF em todo o território, a realização de buscas ativas pelos agentes comunitários de saúde e as campanhas nacionais e locais. Foram observados 15 profissionais de enfermagem em oito salas de vacinas de unidades de ESF, totalizando 106 observações relacionadas aos registros de imunobiológicos, o que pode contribuir para a baixa cobertura vacinal: 49,1\% dos usuários receberam vacinas fora da faixa etária preconizada pelo esquema vacinal; $13,2 \%$ dos usuários receberam o cartão de vacinação após o preenchimento pelo profissional sem o aprazamento de doses ou vacinas; $17,8 \%$ não receberam orientações sobre as vacinas aprazadas na cartão de vacinação; $1,9 \%$ não teve o registro correto da vacina aplicada na cartão de vacinas; $16 \%$ dos profissionais não preencheram corretamente os dados no PNI; e 11,3\% as aplicações foram adiadas pela decisão do profissional de enfermagem. ${ }^{23}$ 
Um outro estudo realizado sobre falha na cobertura vacinal ocorreu no Sergipe (Região Nordeste), onde foi investigada a situação vacinal das crianças menores de dois anos de idade para se verificar o risco de doenças imunopreveníveis. O estudo apontou um alto risco para doenças imunopreveníveis, pois apenas 46,7\% dos municípios sergipanos atingiram a meta vacinal no ano de 2017. Tais resultados alertam para a necessidade de intervenção para modificar o cenário de risco de reintrodução de doenças controladas. ${ }^{24}$

Os dados globais de 2018 apontam que 14\% das crianças não completaram o esquema vacinal da DTP. Dentre estas crianças, $70 \%$ não receberam nenhuma dose da vacina, somando 19,4 milhões de crianças desprotegidas contra estes agravos. Outro dado importante é que 29\% destas crianças vivem na Índia e na Nigéria, locais com precárias condições sociosanitárias e econômicas. Indiscutivelmente, estratégias para se atingir a cobertura vacinal devem ser desenvolvidas a fim de alcançar esta população não vacinada, de modo a sensibilizar a população sobre a importância das imunizações e derrubar as barreiras da recusa da vacina. ${ }^{8}$

Embora a ESF seja o modelo prioritário de atenção à saúde no Brasil, este estudo evidenciou que uma maior cobertura de ESF não impactou necessariamente no alcance das metas desta vacina (acima de 95\%) como preconizado pelo PNI. Os resultados indicam a proposição de novas investigações para se encontrarem as barreiras e facilidades em capitais brasileiras com cobertura de ESF insuficiente e apresentando cobertura vacinal adequada, além das capitais com excelente cobertura de ESF e vacinação muito abaixo do preconizado.

Por sua vez, a atuação da equipe de enfermagem possui um importante papel no PNI por conta de todas as suas ações de gerenciamento, supervisão e execução de imunobiológicos na ESF. ${ }^{3} \mathrm{O}$ processo de trabalho da equipe de saúde na ESF, especialmente dos profissionais de enfermagem, é repleto de desafios constantes em relação às atividades de imunizações, o que exige a programação e o desenvolvimento de ações que resgatem pacientes com atrasos vacinais; assim, torna-se possível atingir as coberturas preconizadas. Neste sentido, ressalta-se a 
relevância do papel da enfermagem de orientar sobre a importância da imunização individual, coletiva, assim como sobre o perigo das notícias falsas. Tais medidas ajudam na garantia da imunização populacional no território em questão. Logo, o enfermeiro como responsável técnico pela sala de vacinas, precisa desenvolver uma atitude proativa por meio de ações educativas da equipe de saúde na ESF diante dos indicadores de coberturas vacinais. . $11,19,23,25$

Em vista desta situação do indicador da cvP em diferentes regiões e capitais do Brasil, desvela-se a necessidade de que todas as capitais compartilhem as ações de saúde exitosas na vacinação. Com certeza, trata-se de um trabalho em rede colaborativa que permite unir esforços para sensibilizar a população para vacinação e implementar estratégias que visem melhorar a hesitação vacinal para se atingir a meta preconizada e, dessa forma, evitar doenças imunopreveníveis e mortalidade infantil.

Quanto às limitações encontradas neste estudo, observa-se a carência de produção científica brasileira sobre o tema para além dos manuais e de boletins epidemiológicos da vigilância em saúde do MS. No que se refere ao DATASUS e ao e-Gestor AB, os mesmos estão sujeitos às atualizações, podendo acarretar mudanças nas informações. Ademais, a confiabilidade dos dados depende do adequado processo de registro eletrônico ou manual nos sistemas locais, o que representa as subnotificações.

\section{Conclusão}

Este trabalho buscou avaliar no cenário brasileiro a cobertura vacinal do imunobiológico Pentavalente em menores de um ano e a cESF, no período de 2014 a 2018. Nenhuma região brasileira, desde 2017, atingiu a meta preconizada de 95\% de cvP, sinalizando a necessidade de se repensarem as estratégias das ações em saúde para vacinação da população. Entre as capitais que atingiram a meta preconizada, em 2018, observou-se Porto Velho, Fortaleza, Natal, Rio de Janeiro e Vitória. Entretanto, as capitais que mostraram a existência de ações em saúde para o aumento deste 
indicador, na comparação de 2014 e 2018, foram Porto Velho, Rio Branco, Fortaleza, Maceió, Natal, Campo Grande, Belo Horizonte, São Paulo, Rio de Janeiro e Curitiba.

Em seis capitais ocorreu a ampliação da cvP e da cESF na comparação de 2014 e 2018: Rio Branco, Fortaleza, Campo Grande, Belo Horizonte, Rio de Janeiro e São Paulo. Entretanto, algumas capitais investiram na ampliação da cESF, ao se compararem os indicadores de 2014 e 2018; são elas: Belém, Palmas, João Pessoa, Recife, Salvador, São Luís, Teresina, Brasília, Cuiabá, e Porto Alegre.

Incontestavelmente, o acesso aos serviços de saúde precisa ser facilitado, devendo a vacinação ser sempre priorizada no planejamento das ações na APS. Dessa forma, evita-se perder oportunidades de vacinar todos usuários que busquem o serviço de saúde, independentemente do local de moradia. A equipe de enfermagem é a responsável pela sala de vacinação e por criar estratégias a nível local para o alcance da sua meta tais como: busca de faltosos, campanha de vacinação, educação em saúde, atividades extramuros, registros adequados nos sistemas de informações, entre outras.

Além disso, o papel das equipes de ESF deve ser revisitado, buscando-se intensificar as ações de prevenção de doenças e promoção de saúde. Todos os membros da equipe devem trabalhar de forma integrada, compartilhada e colaborativa, esclarecendo todas dúvidas da população e estimulando a vacinação. Sem dúvida, de todas possíveis causas para o atraso vacinal e do cenário de cESF apresentadas nesta pesquisa, acredita-se que o Brasil tenha uma longa jornada para retomar o aumento deste indicador de coberturas vacinais e garantir a proteção da população. Dessa forma, haveria uma redução da mortalidade precoce e dos custos em saúde relacionados ao atendimento clínico e internações hospitalares decorrentes dessas doenças imunopreveníveis.

Diante da magnitude dos resultados encontrados, recomenda-se que novos estudos sejam desenvolvidos para verificar in loco as razões e fatores que podem estar interferindo em cada Região e Capital brasileira que expliquem o atraso e hesitação vacinal. Dada a importância do enfermeiro nas imunizações, sugere-se que este profissional esteja à frente de novas pesquisas 
ampliando o conhecimento da Enfermagem sobre este cenário. Já os órgãos públicos devem estar atentos e intensificar as ações em imunizações, assim como combater as causas que têm prejudicado o alcance das metas vacinais.

\section{Referências}

1. Ministério da Saúde (BR). Programa Nacional de Imunizações (PNI). Brasília (DF): Ministério da Saúde; 2015 [acesso em: 2019 nov 20]. Disponível em: https://portalarquivos.saude.gov.br/campanhas/pni/

2. Ballalai I, Bravo F, organizadores. Imunização: tudo o que você sempre quis saber. 3a. ed. Rio de Janeiro: Sociedade Brasileira de Imunizações; 2016 [acesso em 2019 nov 17]. Disponível em: https://sbim.org.br/publicacoes/livros/564-imunizacao-tudo-o-que-voce-sempre-quis-saber

3. Ministério da Saúde (BR). Coberturas vacinais no Brasil: período: 2010 - 2014. Brasília (DF): Ministério da Saúde; 2015 [acesso em 2019 out 24]. Disponível em: https://portalarquivos2.saude.gov.br/images/pdf/2017/agosto/17/AACOBERTURAS-VACINAIS-NO-BRASIL--2010-2014.pdf

4. Waissmann W. Cobertura vacinal em declínio: hora de agir! Vigil Sanit Debate [Internet]. 2018 [cited 2019 Oct 24];6(3):1-3. Available from: https://visaemdebate.incqs.fiocruz.br/index.php/visaemdebate/article/view/1189

5. Nour R. A systematic review of methods to improve attitudes towards childhood vaccinations. Cureus. 2019;11(7):e5067. doi: 10.7759/cureus.5067

6. Sato APS. Qual a importância da hesitação vacinal na queda das coberturas vacinais no Brasil? Rev Saúde Publica. 2018;52:96. doi: 10.11606/s1518-8787.2018052001199

7. Sociedade Brasileira de Imunizações. Vacinas [Internet]. São Paulo: SBIm; 2019 [acesso em 2019 nov 05]. Disponível em: https://familia.sbim.org.br/vacinas

8. Peck M, Gacic-Dobo M, Diallo MS, Nedelec Y, Sodha SS, Wallace AS. Global routine vaccination coverage, 2018. MMWR Morb Mortal Wkly Rep. 2019;68(42):937-42. doi:10.15585/mmwr.mm6842a1

9. Marinelli NP, Carvalho KM, Araújo TME. Conhecimento dos profissionais de enfermagem em sala de vacina: análise da produção científica. Rev UNIVAP. 2015;21(38):26-35. doi: 10.18066/revistaunivap.v21i38.324

10. Fossa AM, Protti AM, Rocha MCP, Horibe TM, Pedroso GER. Conservação e administração de vacinas: a atuação da enfermagem. Saúde Rev. 2015;15(40):85-96. doi: 10.15600/2238-1244/sr.v15n40p85-96

11. Pereira MAD, Lima BC, Donnini DA, Oliveira VC, Gontijo TL, Renno HMS. Gerenciamento de enfermagem em sala de vacinas: desafios e oportunidades. Rev Enferm UFSM [Internet]. 2019 [cited 2019 Nov 
20];9(32):1-18. doi: https://periodicos.ufsm.br/reufsm/article/view/33279

12. Gomes CBS, Gutiérrez AC, Soranz D. Política Nacional de Atenção Básica de 2017: análise da composição das equipes e cobertura nacional da Saúde da Família. Ciênc Saúde Colet. 2020;25(4):1327-38. doi:10.1590/1413-81232020254.31512019

13. Ministério da Saúde (BR). Departamento de Informática do SUS - DataSUS. Brasília (DF): Ministério da Saúde; 2019 [acesso em 2019 dez 30]. Disponível em: http://www2.datasus.gov.br/DATASUS/index.php?area=01

14. Ministério da Saúde (BR). Sistema e-Gestor Atenção Básica. Brasília (DF): Ministério da Saúde; 2019 [acesso em 2019 out 05]. Disponível em: https://egestorab.saude.gov.br

15. BRASIL. Ministério da Saúde, Conselho Nacional de Saúde. Resolução no 466, de 12 de dezembro de 2012. Diretrizes e normas regulamentadoras de pesquisas envolvendo seres humanos. Diário Oficial da União: seção 1, Brasília, DF, n. 112, p. 59-62, 13 jun. 2013.

16. Waldman EA, Sato APS. Trajetória das doenças infecciosas no Brasil nos últimos 50 anos: um contínuo desafio. Rev Saúde Pública. 2016;50:68. doi: 10.1590/S1518-8787.2016050000232

17. Carpiano RM, Fitz NS. Public attitudes toward child undervaccination: a randomized experiment on evaluations, stigmatizing orientations, and support for policies. Soc Sci Med. 2017;185:127-36. doi: 10.1016/j.socscimed.2017.05.014

18. Pereira JPC, Braga GM, Costa GA. Negligência à vacinação: o retorno do sarampo ao Brasil. e-Sci [Internet]. 2019 [acesso em 2019 dez 30];12(1):1-5. Disponível em: https://revistas.unibh.br/dcbas/article/view/2826/pdf

19. Orr D, Baram-Tsabari A, Landsman K. Social media as a platform for health-related public debates and discussions: the Polio vaccine on Facebook. Isr J Health Policy Res. 2016;5:34. doi: 10.1186/s13584-016-0093-4

20. Sanches SHDFN, Cavalcanti AELW. Direito à saúde na sociedade da informação: a questão da fake news e seus impactos na vacinação. Rev Jurídica. 2018;4(53):448-66. doi: 10.6084/m9.figshare.7628969

21. Ministério da Saúde (BR). Recebeu mensagem sobre saúde? Não compartilhe antes de checar [Internet]. Brasília (DF): Ministério da Saúde; 2019 [acesso em 2019 nov 11]. Disponível em: http:/www.blog.saude.gov.br/index.php/servicos/53959-recebeu-mensagem-sobre-saude-nao-compartilheantes-de-checar-se-e-verdade

22. Shende P, Waghchaure M. Combined vaccines for prophylaxis of infectious conditions. Artif Cells Nanomed Biotechnol. 2019;47:696-705. doi: 10.1080/21691401.2019.1576709

23. Dalla Nora TT, Herrera GW, Linch GFC, Paz AA. Registro de dados sobre o uso de imunobiológico e insumos na sala de vacinas. Cogitare Enferm. 2018;23(4):e59274. doi:10.5380/ce.v23i4.56274 
24. Cunha JO, Farias LHS, Góes JAP, Bispo MM, Anjos TS, Silva GM, et al. Classificação de risco de doenças imunopreveníveis e sua distribuição espacial. Cogitare Enferm. 2020;25:e68072. doi: 10.5380/ce.v25i0.68072

25. Dalla Nora TT, Paz AA, Linch GFC, Weis AH, Wachter MZD. Situação da cobertura vacinal de imunobiológicos no período de 2009-2014. Rev Enferm UFSM. 2016;6(4):482-93. doi: 10.5902/2179769221605

Editora Científica Chefe: Cristiane Cardoso de Paula

Editora associada: Graciela Dutra Sehnem

\section{Autor correspondente}

Marilene Lopes Vieira

E-mail: marilenelv991747701@gmail.com

Endereço: Av. Baltazar de Oliveira Garcia, 744, São Sebastião, Porto Alegre, RS, Brasil.

\section{Contribuições de Autoria}

\section{1 - Marilene Lopes Vieira}

Concepção ou desenho do estudo/pesquisa, análise e/ou interpretação dos dados, revisão final com participação crítica e intelectual no manuscrito.

\section{2 - Sabrina Ribeiro Soares}

Análise e/ou interpretação dos dados, revisão final com participação crítica e intelectual no manuscrito.

\section{3 - Lenisa Bernardes dos Santos}

Análise e/ou interpretação dos dados, revisão final com participação crítica e intelectual no manuscrito.

\section{4 - Franciele dos Santos Moreira}

Revisão final com participação crítica e intelectual no manuscrito.

\section{5 - Graciele Fernanda da Costa Linch}

Concepção ou desenho do estudo/pesquisa, análise e/ou interpretação dos dados, revisão final com participação crítica e intelectual no manuscrito.

\section{6 - Adriana Aparecida Paz}

Concepção ou desenho do estudo/pesquisa, análise e/ou interpretação dos dados, revisão final com participação crítica e intelectual no manuscrito.

\section{Como citar este artigo}

Vieira ML, Soares SR, Santos LB, Moreira FS, Linch GFC, Paz AA. Cobertura vacinal da Pentavalente e da Estratégia de Saúde da Família. Rev. Enferm. UfSM. 2021 [Acesso em: Ano Mês Dia]; vol.11 e16: 1-21. DOI:https://doi.org/10.5902/2179769243442 